\title{
Parallel Processing of Nociceptive and Non-nociceptive Somatosensory Information in the Human Primary and Secondary Somatosensory Cortices: Evidence from Dynamic Causal Modeling of Functional Magnetic Resonance Imaging Data
}

\author{
Meng Liang, ${ }^{1}$ André Mouraux, ${ }^{2}$ and Gian Domenico Iannetti ${ }^{1}$ \\ ${ }^{1}$ Department of Neuroscience, Physiology, and Pharmacology, University College London, London WC1E 6BT, United Kingdom, and ${ }^{2}$ Institute of \\ Neuroscience, Université Catholique de Louvain, B-1200 Brussels, Belgium
}

Several studies have suggested that, in higher primates, nociceptive somatosensory information is processed in parallel in the primary (S1) and secondary (S2) somatosensory cortices, whereas non-nociceptive somatosensory input is processed serially from S1 to S2. However, evidence suggesting that both nociceptive and non-nociceptive somatosensory inputs are processed in parallel in S1 and S2 also exists. Here, we aimed to clarify whether or not the hierarchical organization of nociceptive and non-nociceptive somatosensory processing in S1 and S2 differs in humans. To address this question, we applied dynamic causal modeling and Bayesian model selection to functional magnetic resonance imaging (fMRI) data collected during the selective stimulation of nociceptive and non-nociceptive somatosensory afferents in humans. This novel approach allowed us to explore how nociceptive and non-nociceptive somatosensory information flows within the somatosensory system. We found that the neural activities elicited by both nociceptive and non-nociceptive somatosensory stimuli are best explained by models in which the fMRI responses in both S1 and S2 depend on direct thalamocortical projections. These observations indicate that, in humans, both nociceptive and non-nociceptive information are processed in parallel in S1 and S2.

\begin{abstract}
Introduction
The pivotal role of the primary (S1) and secondary (S2) somatosensory cortices in the central processing of both nociceptive and non-nociceptive somatosensory information is well established (Mountcastle, 2005). There is compelling anatomical evidence from primates that $S 1$ and S2 are reciprocally connected through extensive corticocortical projection neurons arising from superficial layers (I-III) (Friedman et al., 1980; Burton and Carlson, 1986; Pons and Kaas, 1986; Kandel et al., 2010). Importantly, both S1 and S2 receive direct projections from multiple thalamic nuclei such as the ventral posterior nucleus (VP) (including ventral posterior lateral and ventral posterior medial nuclei), the ventral posterior inferior nucleus (VPI), and the centrolateral nucleus (CL) (Jones and Leavitt, 1974; Burton and Jones, 1976;
\end{abstract}

Received Nov. 26, 2010; revised April 15, 2011; accepted April 19, 2011.

Author contributions: M.L., A.M., and G.D.I. designed research; M.L., A.M., and G.D.I. performed research; M.L. analyzed data; M.L., A.M., and G.D.I. wrote the paper.

This work was supported by a Biotechnology and Biological Sciences Research Council grant. G.D.I. is supported by a Royal Society University Research Fellowship. We thank Dr. Li Hu for his insightful comments on this study.

The authors declare no competing financial interests.

Correspondence should be addressed to Dr. Giandomenico lannetti, Department of Neuroscience, Physiology, and Pharmacology, University College London, Medical Sciences Building, Gower Street, London WC1E 6BT, United Kingdom. E-mail: g.iannetti@ucl.ac.uk.

DOI:10.1523/JNEUROSCI.6207-10.2011

Copyright $\odot 2011$ the authors $\quad 0270-6474 / 11 / 318976-10 \$ 15.00 / 0$
Friedman and Murray, 1986; Krubitzer and Kaas, 1992; Jones, 1998). All these nuclei relay nociceptive and non-nociceptive somatosensory inputs to both S1 and S2 (Gingold et al., 1991; Shi et al., 1993; Stevens et al., 1993). However, whether nociceptive and non-nociceptive somatosensory inputs are processed differently in S1 and S2 remains a matter of debate (Allison et al., 1989a; Pons et al., 1992; Rowe et al., 1996; Bushnell et al., 1999; Karhu and Tesche, 1999; Ploner et al., 1999), although recent evidence has suggested that, in higher primates including humans, the hierarchy of their cortical processing in S1 and S2 may be fundamentally different (Ploner et al., 1999; Apkarian et al., 2005).

In lower primates, several studies have shown that nonnociceptive somatosensory information is transmitted from the thalamus to both S1 and S2 via segregated thalamocortical pathways, thus indicating a parallel processing of non-nociceptive somatosensory input in S1 and S2 (Garraghty et al., 1991; Turman et al., 1992). In contrast, the processing of non-nociceptive somatosensory input in higher primates and humans remains, at present, a matter of debate (Rowe et al., 1996; Iwamura, 1998). A number of studies have suggested that non-nociceptive somatosensory input is processed serially from S1 to S2 (i.e., from the thalamus to S1, and then from S1 to S2) (Allison et al., 1989a,b; Pons et al., 1992; Hari et al., 1993; Mima et al., 1998; Schnitzler et al., 1999; Inui et al., 2004; Ploner et al., 2009). This difference in 
hierarchical organization has been interpreted as the result of an evolutionary shift from a phylogenetically older parallel organization to a more recent serial organization of somatosensory processing in S1 and S2 (Mountcastle, 2005). However, a small number of studies have suggested the opposite: that, even in higher primates, non-nociceptive somatosensory input is processed in parallel in S1 and S2 (Rowe et al., 1996; Zhang et al., 1996, 2001; Karhu and Tesche, 1999).

In contrast with the large body of evidence investigating the serial versus parallel processing of non-nociceptive somatosensory input in S1 and S2 in higher primates, only few studies, all relying on magnetoencephalography, have suggested a parallel processing of nociceptive somatosensory input in S1 and S2 in humans (Ploner et al., 1999; Kanda et al., 2000).

Hence, additional evidence is needed to clarify whether in humans (1) the cortical processing of non-nociceptive input in S1 and S2 is serial or parallel, and (2) whether the organization of nociceptive and non-nociceptive somatosensory processing in S1 and S2 truly differs. In the present study, we addressed these two questions by applying dynamic causal modeling (DCM) and Bayesian model selection (BMS) to functional magnetic resonance imaging (fMRI) data collected during the selective stimulation of $\mathrm{A} \beta$ (non-nociceptive) and A $\delta$ (nociceptive) somatosensory afferents in humans. This novel approach allowed exploring a specific aspect of the physiological information contained in the blood oxygen leveldependent (BOLD) fMRI time series (i.e., how nociceptive and non-nociceptive somatosensory information flows within the somatosensory system).

\section{Materials and Methods}

Participants. Fourteen healthy right-handed volunteers took part in the study (six females and eight males; aged 20-36 years). All participants gave written informed consent, and the experimental procedures were approved by the local ethics committee.

Experimental design and data acquisition. Lights in the scanner room were dim. While lying in the scanner, participants received stimuli of four different sensory modalities: nociceptive somatosensory, non-nociceptive somatosensory, auditory, and visual, and all stimuli were delivered to or around the participant's right side (Mouraux et al., 2011). The brain responses elicited by auditory and visual stimuli were not analyzed and, hence, are not reported in the present study. Nociceptive somatosensory stimuli were pulses of radiant heat ( $5 \mathrm{~ms}$ duration) generated by an infrared neodymium yttrium aluminum perovskite (Nd:YAP) laser (wavelength, $1.34 \mu \mathrm{m}$; ElEn Group). The laser beam was transmitted through an optic fiber, and focusing lenses were used to set the diameter of the beam at target site to $\sim 7 \mathrm{~mm}$. The energy of the stimulus ( $3 \pm 0.5$ $J)$ was set to elicit a clear painful pinprick sensation, related to the selective activation of A $\delta$ skin nociceptors (Bromm and Treede, 1984). The stimulus was applied to the dorsum of the right foot, within the sensory territory of the superficial peroneal nerve. To prevent fatigue or sensitization of nociceptors, the laser beam was manually displaced by $\sim 2 \mathrm{~cm}$ after each stimulus. Non-nociceptive somatosensory stimuli were constant current square-wave electrical pulses ( 1 ms duration; DS7A; Digitimer), delivered through a pair of skin electrodes $(1 \mathrm{~cm}$ interelectrode distance) placed at the right ankle, over the superficial peroneal nerve. For each participant, stimulus intensity $(6 \pm 2 \mathrm{~mA})$ was adjusted to elicit a nonpainful paresthesia in the sensory territory of the nerve. The intensity of electrical stimulation was above the electrical activation threshold of $\mathrm{A} \beta$ fibers (which convey innocuous non-nociceptive sensations) but well below the electrical activation threshold of nociceptive $\mathrm{A} \delta$ and $\mathrm{C}$ fibers (Burgess and Perl, 1967; Mouraux et al., 2010), and never elicited a painful percept. Visual stimuli consisted of a bright white disk $\left(\sim 9^{\circ}\right.$ viewing angle) displayed on the projection screen, above the right foot, for $100 \mathrm{~ms}$. Auditory stimuli were loud $(65 \mathrm{~dB})$, right-lateralized $800 \mathrm{~Hz}$ tones ( 0.5 left/right amplitude ratio; $50 \mathrm{~ms}$ duration; $5 \mathrm{~ms}$ rise and fall times), delivered binaurally through custom-built pneumatic earphones bored into a set of low-profile ear defenders (Mayhew et al., 2010).

The fMRI experiment consisted of a single acquisition, divided into four successive runs. Each run consisted of a stimulation period $(\sim 8 \mathrm{~min}$ duration), followed by a rating period ( $\sim 2$ min duration). During the stimulation period, each type of stimulus was delivered 8 times (4 stimulus modalities $\times 8=32$ stimuli/period). All stimuli were delivered in a pseudorandom order, such that stimuli of the same sensory modality were not delivered consecutively more than twice. The interstimulus interval (ISI) was $10,13,16$, or 19 s. For each stimulus modality, each ISI was used 8 times ( 4 stimulus modalities $\times 4$ ISI $\times 8=128$ stimuli in total). The order of ISIs was pseudorandomized, such that the same ISI was not used consecutively more than twice. Throughout the stimulation sequence, participants were instructed to fixate a white cross $\left(\sim 1.5^{\circ}\right.$ viewing angle) displayed at the center of the screen. During the rating period, participants were asked to rate the saliency of each stimulus modality. This was done by adjusting the position of a cursor on four consecutively displayed visual-analog scales, labeled "laser," "electric," "visual," and "auditory." Each scale was displayed for 9 s. For each rating, the position of the cursor was transformed into a numerical value between 0 and 10. Left and right extremities of the scales were labeled "not salient" and "extremely salient." The order of presentation of the four scales was randomized across blocks. Stimulus saliency was explained to each participant as "the ability of the stimulus to capture attention" (Mouraux and Iannetti, 2009). Therefore, this behavioral feedback was expected to integrate several factors such as stimulus intensity, frequency of appearance, novelty, and its potential relevance to behavior. Several studies have shown that human judgments of saliency correlate well with predicted models of saliency (Kayser et al., 2005).

Functional MRI data were acquired using a 3T Varian-Siemens wholebody magnetic resonance scanner (Oxford Magnet Technology). A head-only gradient coil was used with a birdcage radiofrequency coil for pulse transmission and signal reception (a whole-brain gradient-echo time; 41 contiguous 3.5-mm-thick slices; field of view, $192 \times 192 \mathrm{~mm}$; matrix, $64 \times 64$; with a repetition time of 3 s over 740 volumes, resulting in a total scan time of $37 \mathrm{~min})$. At the end of the experiment, a T1weighted structural image (1-mm-thick axial slices; in-plane resolution, $1 \times 1 \mathrm{~mm}$ ) was acquired for spatial registration and the anatomical overlay of the functional data.

Data preprocessing. The MRI data were analyzed using SPM8 (Wellcome Trust Centre for Neuroimaging, London, UK; http://www.fil. ion.ucl.ac.uk/spm/). Data preprocessing included the following steps. For each individual dataset, the first four volumes were discarded to allow for signal equilibration. The remaining $736 \mathrm{fMRI}$ volumes were spatially realigned, normalized to the Montreal Neurological Institute (MNI) space using the unified normalization-segmentation procedure of SPM8, resampled to $3 \times 3 \times 3 \mathrm{~mm}^{3}$ voxel size, and spatially smoothed with an isotropic $8 \mathrm{~mm}$ full-width at half-maximum Gaussian kernel. Finally, the time series from each voxel were high-pass filtered $(1 / 128 \mathrm{~Hz}$ cutoff) to remove low-frequency noise and signal drifts.

Regions of interest selection. For each participant, first-level statistical parametric maps were obtained using a general linear model with regressors modeling the occurrence of each of the four types of stimuli (nociceptive somatosensory, non-nociceptive somatosensory, auditory, and visual) and their corresponding temporal and dispersion derivatives. Additional regressors were defined using the head motion parameters estimated during the fMRI volumes realignment in preprocessing. To identify the brain areas responding to both nociceptive and nonnociceptive somatosensory stimulation, a conjunction analysis was performed using the nociceptive and non-nociceptive activation maps obtained for each individual, as implemented in SPM8 (Price and Friston, 1997; Friston et al., 1999, 2005; Caplan and Moo, 2004; Nichols et al., 2005). Group-level conjunction maps were obtained as a second-level analysis across participants. These conjunction maps were finally used to define the three regions of interest (ROIs) used for DCM: the thalamus, S1, and S2, all contralateral to the stimulated side (i.e., the three brain structures concerned with our hypothesis testing). Defining the ROIs using the conjunction maps was justified by the fact that only models comprising exactly the same voxels can be validly compared using BMS 
A

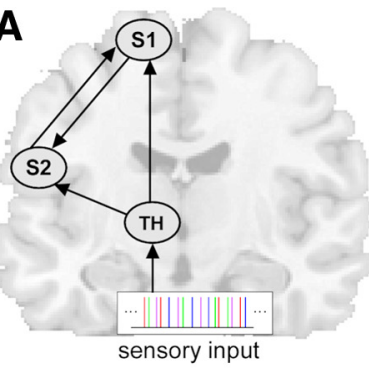

E

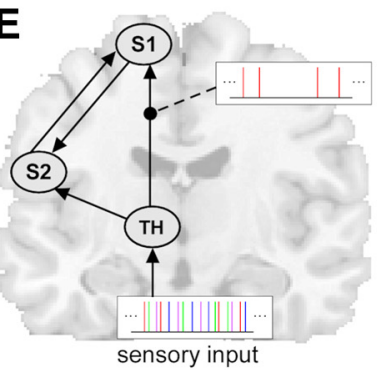

I

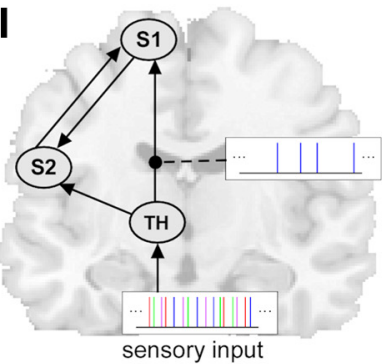

M

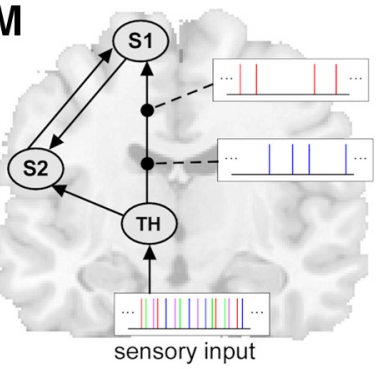

B

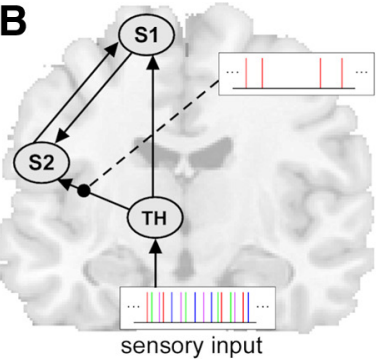

$\mathbf{F}$

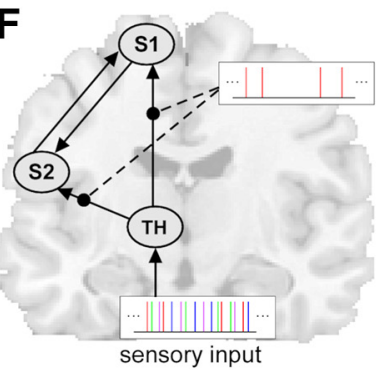

J

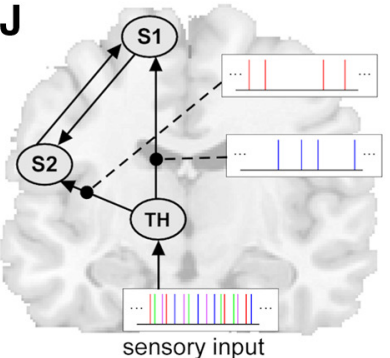

$\mathbf{N}$

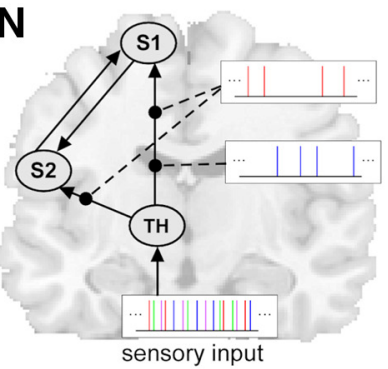

C

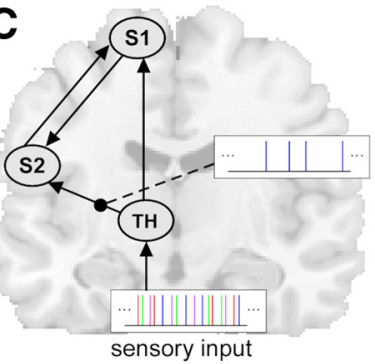

G

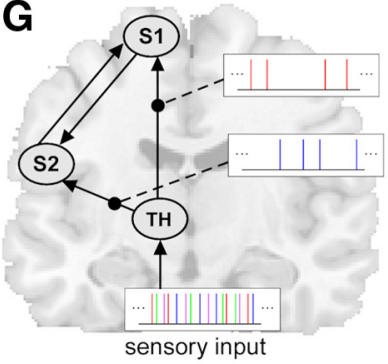

K

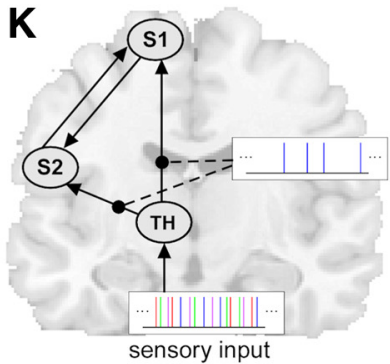

0

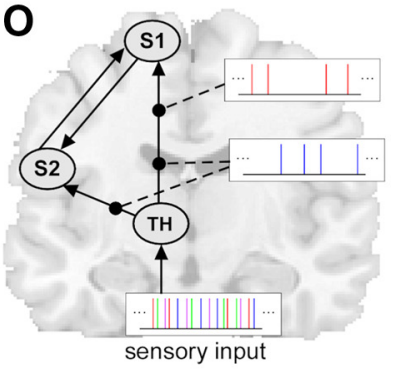

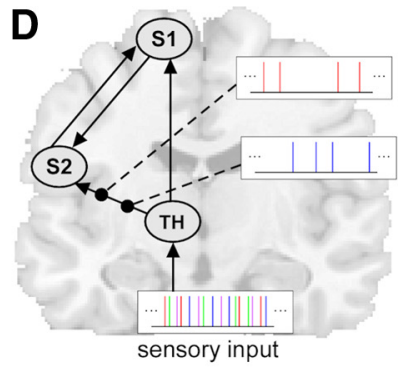

H

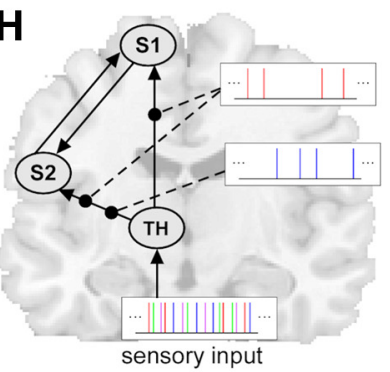

L

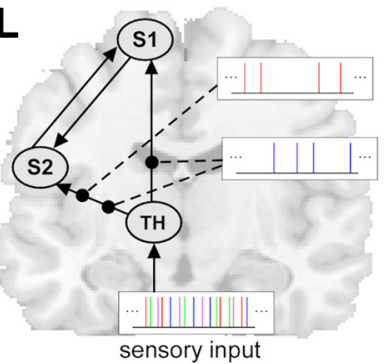

P

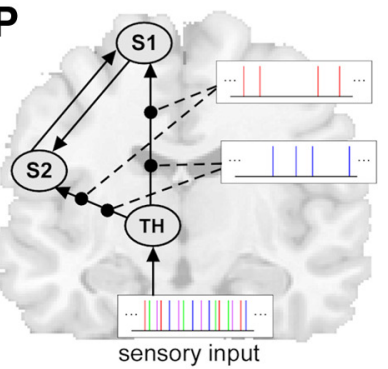

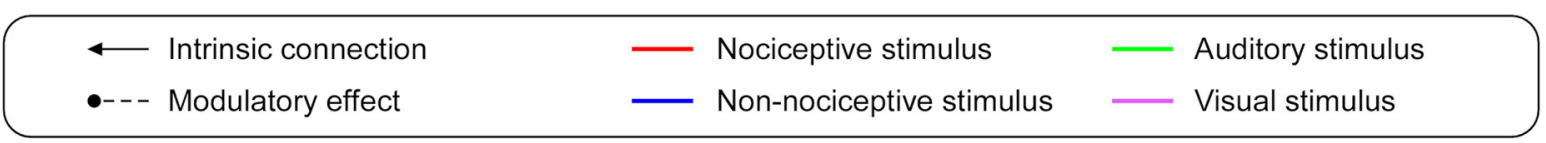

Figure 1. Structures of the 16 DCM model families $(A-P)$. In all model families, four intrinsic connections were defined. These intrinsic connections are indicated by the black lines with arrows, and the arrows indicate the direction of the connectivity. The 16 model families differ in terms of how the connections between the thalamus and $\mathrm{S}$ and between the thalamus and $\mathrm{S} 2$ are modulated (i.e., whether each of the two connections are modulated by non-nociceptive stimuli, by nociceptive stimuli, by both stimuli, or by neither of them). These modulations are indicated by the black dashed lines. The color of the vertical lines at the end of the dashed lines indicates the modality of the stimuli that exert the modulatory effect. Each model family contains 16 single models (data not shown) that differ in how the connections between $\mathrm{S} 1$ and S2 are modulated. Therefore, 256 models in total (16 models $\times 16$ families) were defined in the present study. The thalamus was set as the receiving area, and the driving input to the thalamus was formed by all stimuli regardless of their modality. These stimuli are represented by vertical lines with different colors indicating different sensory modalities (red, nociceptive; blue, non-nociceptive; green, auditory; purple, visual). S1, Primary somatosensory cortex; S2, secondary somatosensory cortex; TH, thalamus.

(Stephan et al., 2010). Importantly, our previous study has shown that, in these three brain structures, nociceptive and non-nociceptive somatosensory stimuli elicit spatially indistinguishable responses (Mouraux et al., 2011). Therefore, the ROIs defined by the conjunction maps included the bulk of the BOLD responses elicited by both types of somatosensory stimuli.

ROIs were defined as follows. (1) The response local maximum in the thalamus, S1, and S2 was identified in the group-level conjunction maps. (2) Starting from the group local maximum, the nearest local maximum of each ROI was identified in the conjunction maps of each participant.
(3) Single-subject ROIs were constructed by including all the 19 voxels contained within a sphere (radius, $5 \mathrm{~mm}$ ) centered over each subject's local maximum. For each participant and ROI, a BOLD time course was obtained using the first eigenvector of the time series of all the voxels contained within this ROI, adjusted for the $F$ contrast of effects of interests to remove the head motion related confound, as implemented in SPM8.

Dynamic causal modeling. DCM analysis of BOLD fMRI signal is an approach that has been introduced to estimate the effective connectivity between different brain areas (Friston et al., 2003) and is receiving increasing 

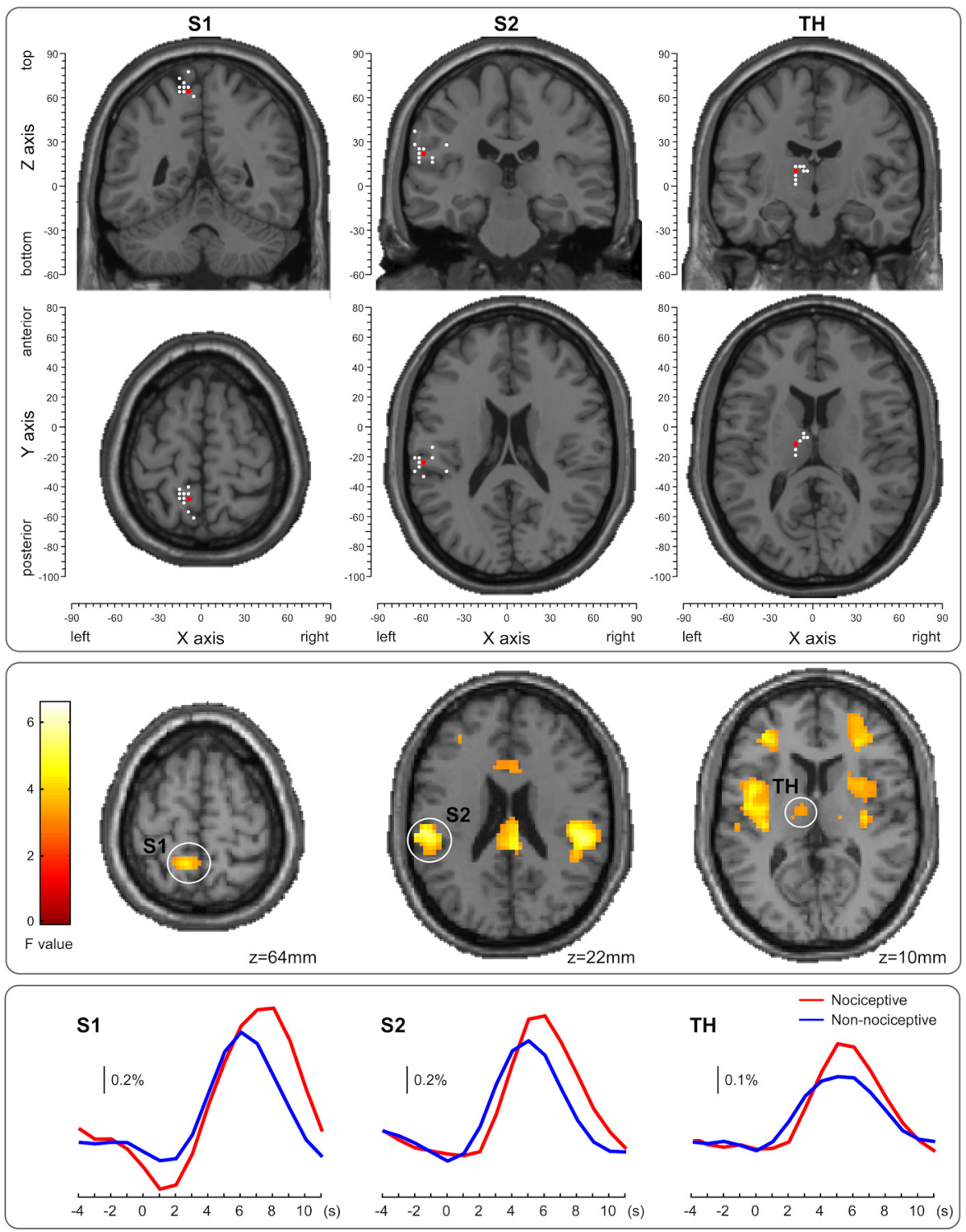

Figure 2. The ROIs used in the DCM analysis: contralateral S1 (left column), contralateral S2 (middle column), and contralateral thalamus (TH) (right column). The locations of the maximally activated voxels across the group (red dots, top panel) were selected from the contralateral (left) hemisphere based on the conjunction map of the responses elicited by nociceptive and nonnociceptive stimuli, thresholded at $p<0.001$ and cluster size of $>10$ voxels (middle panel). The ROls of each participant were formed by the voxels contained within a sphere of $5 \mathrm{~mm}$ radius, centered at the maximally activated voxel (white dots, top panel) nearest to the corresponding group maxima, based on the individual conjunction map thresholded at $p<0.05$ and cluster size of $>10$ voxels. The locations of the group maxima (red dots) and individual maxima (white dots) are superimposed on axial and coronal structural MRIs from the MNI template (top panel), selected from the location of the group maxima. Coordinates of both individual and group maxima of activation are reported in Table 1. The bottom panel shows the BOLD time courses of the responses elicited by both nociceptive and non-nociceptive stimuli in each ROI.

interest (Friston et al., 2007; Kiebel et al., 2007; David et al., 2008; Marreiros et al., 2008; Stephan et al., 2008, 2010; Daunizeau et al., 2009; David, 2009; Friston, 2009a,b; Roebroeck et al., 2009a,b; Schuyler et al., 2010). In DCM, the brain is considered as a dynamic system driven by external perturbations (e.g., the occurrence of an experimental stimulus). Compared with other methods for analyzing effective connectivity of fMRI data such as Granger causal mapping (GCM) (Goebel et al., 2003) or structural equation modeling (McIntosh and Gonzalez-Lima, 1994; Büchel and Friston, 1997), an important advantage of DCM is that the effective connectivity between brain areas is modeled directly at the hidden neural level using an evolution equation, and that the link between neural activity and the hemodynamic BOLD fMRI response is modeled and estimated explicitly by an observation equation (Friston et al., 2003; Daunizeau et al., 2009; Stephan et al., 2010). Therefore, whereas in GCM the effective connectivity is based on the temporal precedence of BOLD signals and can thus easily be contaminated by differences in the hemodynamic response function (HRF) across different brain areas, the inference on brain connectivity obtained by DCM is less affected by the HRF variability and thus yields more accurate results (David et al., 2008). In addition, DCM performed in a bilinear form (Friston et al., 2003) allows modeling not only the effective connectivity between brain areas but also the changes in the effective connectivity caused by experimental perturbations (e.g., the occurrence of a stimulus) (Friston et al., 2003; Stephan et al., 2010). Importantly, DCM is not an exploratory technique [i.e., a technique used to look for connected brain areas by searching the entire brain, such as GCM (Goebel et al., 2003) and psychophysiological interactions (Friston et al., 1997)]. Instead, DCM is a hypothesis-driven technique [i.e., a technique used to test for a specific set of hypotheses, defined a priori (see, in our case, Fig. 1)]. For this reason, DCM is usually combined with BMS (Penny et al., 2004, 2010; Stephan et al., 2009) to test which model or which family of models (i.e., which physiological hypothesis) provides the most likely explanation of the observed data. The combination of DCM and BMS has been validated in two previous studies (David et al., 2008; Schuyler et al., 2010). Using simultaneously recorded electroencephalography (EEG) and fMRI data obtained in rats, David et al. (2008) found that the results of DCM and BMS were consistent with the results of direct functional coupling estimated from intracerebral EEG. Furthermore, Schuyler et al. (2010) reported a high consistency of the estimation of DCM parameters across repeated fMRI acquisitions. DCM combined with BMS has been already applied successfully to test competing hypotheses in other fields of neuroscience, such as to investigate the interhemispheric integration of visual processing, and the cortical interactions related to reading and speech processing (Stephan et al., 2007; Leff et al., 2008; Seghier and Price, 2010).

In the present study, bilinear DCM was performed using the time courses of the three ROIs defining the thalamus, S1, and S2 contralateral to the stimulated side. The bilinear DCM is featured by three different sets of parameters (Friston et al., 2003): (1) intrinsic parameters reflecting the latent connectivity between brain regions in the absence of experimental perturbations (e.g., the occurrence of a sensory stimulus), (2) modulatory parameters reflecting the changes in the intrinsic connectivity caused by experimental perturbations, (3) input parameters reflecting the driving influence on brain regions by external perturbations. These parameters constitute a measure of the tightness of temporal coupling, that is, if a brain area " $A$ " is strongly connected to another brain area "B," neural activity of brain area A will induce a fast change in the neural activity of brain area B (Friston et al., 2003).

In the present study, we hypothesized that the external perturbation generated by sensory stimulation enters our model in the thalamus contralateral to the stimulated side, and we explored the effective connectivity in the pathways connecting the thalamus, S1, and S2. Based on previous knowledge (Apkarian et al., 2005; Kandel et al., 2010), four 
intrinsic connections were defined (Fig. 1): unidirectional connections from the thalamus to $\mathrm{S} 1$, unidirectional connections from the thalamus to S2, and bidirectional connections between S1 and S2. Our hypothesis testing relies on the modulatory parameters (see above), which reflect whether or not the connectivity changes according to the type of external perturbation, that is, whether or not the connectivity from the thalamus to $\mathrm{S} 1$ and from the thalamus to S2 differs during the processing of nociceptive and non-nociceptive somatosensory input. As there are four intrinsic connections in our model, and as each intrinsic connection is associated with four different possible modulatory configurations (i.e., whether a given connection is modulated by nociceptive stimuli, nonnociceptive stimuli, both, or neither), 256 models in total $\left(4^{4}=256\right.$ models) were examined. Because we aimed to test whether the processing toward S1 and S2 is parallel or serial, these 256 models were grouped into 16 different model families according to the modulatory configuration of the connections from the thalamus to S1 and from the thalamus to S2 (Fig. 1). Therefore, the model families differed only in terms of how the connections from the thalamus to $\mathrm{S} 1$ and from the thalamus to $\mathrm{S} 2$ are modulated by nociceptive and non-nociceptive stimuli, and the models within a given family differed only in terms of how the connections between S1 and S2 are modulated. By this partitioning of model space, 16 model families were formed, each containing 16 models (Fig. 1). As the brain responses modeled by DCM are treated as the result of perturbations caused by external stimuli, a driving input and a receiving region must be defined. Because the sensory information belonging to all modalities projects onto the thalamus, from where it is relayed to the cortex, the stimuli belonging to the four different sensory modalities were defined as the driving input, whereas the thalamus was defined as the receiving region. The construction and estimation of the 256 models were performed on each individual dataset, resulting in a total of 3072 models (256 models $\times 12$ participants).

Bayesian model selection. The 16 model families were compared using BMS. BMS uses a Bayesian framework to calculate the model evidence for each model. The model evidence represents a trade-off between the goodness of fit and the complexity of the model, namely the number of parameters defining the model (Penny et al., 2004; Stephan et al., 2010). Here, the model evidence was estimated using the negative free energy, a measure that has been shown to be both more robust and more sensitive compared with the commonly used Akaike information and Bayesian information criteria (Stephan et al., 2009). BMS can be implemented using either fixed-effect analysis [i.e., assuming that the model structure is fixed across participants (FFX BMS)] or random-effect analysis [i.e., assuming that the model structure might vary across participants (RFX BMS)]. RFX BMS was used in the present study, as it treats each model as a random variable and is thus more robust to the presence of outliers than FFX BMS (Stephan et al., 2009). Based on the estimated model evidence of each model, RFX BMS calculates the exceedance probability (i.e., the probability of each model being more likely than any other model). The model with the highest exceedance probability was considered as the best model. When comparing model families, all models within a family were averaged using Bayesian model averaging (BMA) and the exceedance probabilities were calculated for each model family (Penny et al., 2010). An average model of the winning family was also obtained at group and single-subject level. In the present study, RFX BMS was performed on the 16 model families (to determine the best model family) as well as on the 256 single models (to determine the best single model). Once the best model family and the best single model were determined, the modulatory effect of nociceptive and non-nociceptive inputs on the connections between the thalamus and S1 and S2 were further compared across participants, using a paired $t$ test, to assess whether these two somatosensory modalities modulated the two connections differently.

\section{Results}

\section{Behavioral data}

All subjects described nociceptive laser stimuli as painful and pricking, whereas non-nociceptive electrical stimuli elicited a mild paresthesia that was never described as painful. The average ratings of stimulus saliency were as follows: nociceptive somato-
Table 1. MNI coordinates of ROIs at group level and individual level

\begin{tabular}{llll}
\hline & S1 & S2 & Thalamus \\
\hline Group & $-9,-49,64$ & $-57,-25,22$ & $-12,-13,13$ \\
S01 & $-15,-46,64$ & $-60,-28,22$ & $-12,-16,7$ \\
S03 & $-9,-46,64$ & $-60,-25,19$ & $-3,-7,10$ \\
S04 & $-12,-46,67$ & $-60,-28,25$ & $-6,-4,13$ \\
S05 & $-12,-49,70$ & $-51,-22,19$ & $-12,-13,13^{a}$ \\
S07 & $-9,-58,67$ & $-63,-31,37$ & $-12,-13,1$ \\
S08 & $-15,-43,73$ & $-63,-22,28$ & $-12,-13,13^{a}$ \\
S09 & $-6,-61,61$ & $-60,-22,16$ & $-12,-19,10$ \\
S10 & $-12,-52,64$ & $-57,-34,25$ & $-12,-16,4$ \\
S11 & $-15,-49,64$ & $-51,-13,16$ & $-12,-13,10$ \\
S12 & $-9,-49,64^{a}$ & $-60,-28,19$ & $-12,-13,13^{a}$ \\
S13 & $-9,-40,79$ & $-42,-31,31$ & $-9,-10,13$ \\
S14 & $-15,-49,67$ & $-60,-22,19$ & $-6,-7,10$ \\
\hline
\end{tabular}

${ }^{a}$ No activation was observed at the threshold of $p<0.05$ (uncorrected) and cluster size $>10$ voxels; therefore, the group ROI was used for these four single-subject ROls.

sensory, $6.1 \pm 2.2$; non-nociceptive somatosensory, $5.2 \pm 2.2$; auditory, $5.1 \pm 3.0$; visual, $5.0 \pm 1.7$. The average ratings of saliency were not significantly different across modalities (repeated-measures ANOVA: $F_{(3,39)}=0.75, p=0.53$ ).

\section{General linear model analysis, conjunction analysis, and ROI selection}

At single-subject level, all but two participants showed reliable BOLD responses to nociceptive and non-nociceptive somatosensory stimulation ( $p<0.05$, uncorrected; cluster size, $>10$ voxels) and, hence, a corresponding conjunct activation ( $p<0.05$, uncorrected; cluster size, $>10$ voxels). The two participants who did not show any response to non-nociceptive stimulation, and consequently no conjunct activation, were discarded from additional analyses.

A threshold of $p<0.001$ (uncorrected) and cluster size of $>10$ voxels was applied to the group-level conjunction map. The areas jointly activated by both nociceptive and non-nociceptive somatosensory stimuli included the contralateral $\mathrm{S} 1$, and bilateral thalamus, S2, insula, temporal superior lobe, inferior frontal lobe, supplementary motor area, mid-cingulate cortex, and anterior cingulate cortex. Figure 2, middle panel, shows the conjunct responses in the thalamus, S1, and S2 in the hemisphere contralateral to the stimulated side, which were used to build the DCM models. The BOLD time courses of the responses elicited by both nociceptive and non-nociceptive stimuli in each ROI are shown in the bottom panel of Figure 2.

Table 1 and Figure 2, top panel, show the coordinates of the maxima of the three ROIs at group level and the coordinates of the nearest local maxima of each ROI in each participant. The contralateral thalamus of three participants (S05, S08, and S12) and the contralateral S1 of one participant (S12) did not reach the significance threshold of $p<0.05$ and the cluster threshold of size $>10$ voxels. Therefore, the coordinates of the group-level maxima were used to define these single-subject ROIs.

\section{DCM and BMS}

The group-level exceedance probabilities of all 16 model families are shown in the top left panel of Figure 3. One single family (family P) displayed an exceedance probability (0.79) that was far greater than the exceedance probabilities of all other families. Indeed, the second highest exceedance probability, in the families $\mathrm{H}$ and $\mathrm{N}$, was 0.038 . Family $\mathrm{P}$ included all models in which both nociceptive and non-nociceptive somatosensory inputs modu- 

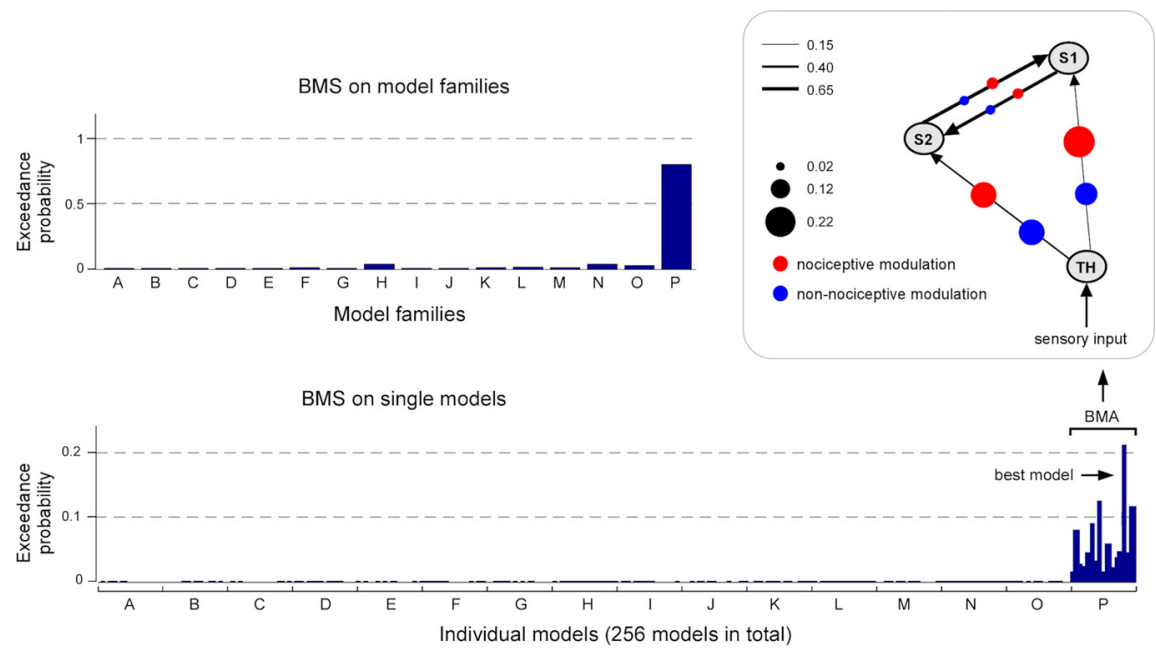

Figure 3. The results of the Bayesian model selection (BMS). Top left panel, The exceedance probabilities of all 16 model families (A-P) showed that the model family $\mathrm{P}$ (in which both connections from the thalamus to $\mathrm{S} 1$ and to $\mathrm{S} 2$ are modulated in both nociceptive and non-nociceptive processing) exceeds by far those of all the other model families. Bottom panel, The exceedance probabilities of all single models (sorted according to model family) showed that the models in family $\mathrm{P}$ had always higher exceedance probabilities and that, within family $\mathrm{P}$, one model seemed to outperform the other models (i.e., it was the best model). Top right panel, Structure of the average model of the winning family P. The black lines with arrows represent the intrinsic connections between brain areas and the thickness of each line indicates the mean strength of each intrinsic connection across participants. The size of the red and blue dots on each connection represents the magnitude of the modulatory effect of nociceptive or non-nociceptive stimulation, respectively. This structure shows that the two forward connections from the thalamus to $S 1$ and to $\$ 2$ were strongly modulated by both nociceptive and non-nociceptive somatosensory inputs, whereas the two reciprocal connections between $\mathrm{S} 1$ and $\mathrm{S} 2$ were only weakly modulated by both types of inputs.

late, in parallel, the connectivity both from the thalamus to $\mathrm{S} 1$ and from the thalamus to S2.

The group-level exceedance probabilities of each of the 256 single models (sorted according to families from A to P) are shown in the bottom panel of Figure 3. All 16 models of the best family (family P) had higher exceedance probabilities $(>0.01)$ than the models belonging to all the other families $(<0.002)$. The structure of the average model of the family $\mathrm{P}$, obtained by averaging all 16 models in this family using BMA, is shown in the top right panel of Figure 3, together with the corresponding estimated parameters. The average model structure showed that the two forward connections from the thalamus to $\mathrm{S} 1$ and from the thalamus to $\mathrm{S} 2$ were strongly modulated by both nociceptive and non-nociceptive somatosensory inputs, whereas the two reciprocal connections between S1 and S2 were only weakly modulated by both types of external perturbations. Among all 256 single models, one model (Fig. 4, model 14, showing an exceedance probability of 0.21 ) outperformed all other models (the exceedance probability of the second best model was 0.11) (Figs. 3,4). Interestingly, the structure of this model indicated that the connection from S2 to S1 was only modulated by nociceptive inputs, indicating a stronger effective connectivity from S2 to S1 in response to nociceptive stimulation.

Using this best model (Fig. 4, model 14), we further compared the modulatory effect of nociceptive and non-nociceptive inputs on the two connections from the thalamus to $\mathrm{S} 1$ and from the thalamus to S2. Paired $t$ tests revealed no significant difference in the modulatory effects of nociceptive and non-nociceptive stimuli on either of these two connections (connection from the thalamus to S1: $p=0.44, t_{(11)}=0.79$; connection from thalamus to $\left.\mathrm{S} 2: p=0.93, t_{(11)}=-0.09\right)$. The same comparison was also performed on the average model of the best family (Fig. 3, family $\mathrm{P})$, yielding a similar result, without significant differences in the modulatory effects of nociceptive and non-nociceptive stimuli on any connection (connection from the thalamus to $\mathrm{S} 1: p=$
$0.42, t_{(11)}=0.84$; connection from the thalamus to S2: $\left.p=0.92, t_{(11)}=-0.10\right)$.

\section{Discussion}

In the present study, we applied DCM and BMS to BOLD fMRI data to test whether, in humans, (1) the cortical processing of non-nociceptive somatosensory input in S1 and S2 is serial or parallel, and (2) whether the organization of nociceptive and non-nociceptive somatosensory processing in S1 and S2 differs. The possible models of brain connectivity were organized in 16 different families according to how the connections from the thalamus to $\mathrm{S} 1$ and from the thalamus to S2 contributed to the BOLD responses elicited by nociceptive and non-nociceptive somatosensory stimuli. BMS showed a clear preference for a family of models in which both nociceptive and non-nociceptive somatosensory inputs modulate in parallel the pathways from the thalamus to $S 1$ and from the thalamus to S2. Therefore, our results indicate (1) that the cortical processing of non-nociceptive somatosensory input in S1 and S2 is parallel, and (2) that this parallel organization of the flow of sensory information in S1 and S2 is similar for nociceptive and nonnociceptive somatosensory processing.

\section{Parallel processing of non-nociceptive input in S1 and S2}

Our results indicate that non-nociceptive somatosensory input is processed in parallel from the thalamus to S1 and from the thalamus to S2 (Figs. 3, 4). This finding is in contradiction to the evidence supporting the notion that, in human and nonhuman higher primates, non-nociceptive somatosensory input is processed serially from the thalamus to S1 and then from S1 to S2 (Allison et al., 1989a,b; Pons et al., 1992; Hari et al., 1993; Mima et al., 1998; Schnitzler et al., 1999; Mountcastle, 2005).

However, it is important to emphasize that the evidence in favor of a serial organization of the cortical processing of nonnociceptive input is not unequivocal. Pons et al. (1992) observed that, in higher primates, the selective ablation of the hand representations in S1 leads to a reduction of the response to nonnociceptive somatosensory stimuli in S2. The observation that the response elicited in S2 is dependent on the presence of an intact $\mathrm{S} 1$ has been interpreted as a strong indication in favor of a serial processing of non-nociceptive input from S1 to S2. However, this observation does not necessarily imply that nonnociceptive somatosensory input reaching S2 is relayed through $\mathrm{S} 1$. Indeed, an alternative interpretation could be that the functional state of S2 is dependent on the functional state of S1, through intrinsic connections between the two areas [i.e., S1 could exert a control on the excitability of S2 neurons (Turman et al., 1992)]. Hence, the ablation of S1 could reduce the responsiveness of $\mathrm{S} 2$ to non-nociceptive inputs originating directly from the thalamus, by removing a background facilitatory influence of S1 on the neural activity in S2 (Turman et al., 1992). Our results, suggesting a direct processing of non-nociceptive somatosensory input from the thalamus to S2 (Fig. 3), as well as the existence of significant intrinsic connectivity between S1 and S2 (Fig. 4), sup- 
port this alternative interpretation. Strong intrinsic connections between S1 and S2 are also compatible with the observation that transcranial magnetic stimulation over S1 can disrupt tactile discrimination in humans not only through a direct effect on the processing of tactile input in S1 but also through an indirect effect on the processing of tactile input in S2 (Cohen et al., 1991; Knecht et al., 2003; Hannula et al., 2008). Finally, it is important to mention that the observations suggesting that the responses elicited in S2 are relayed in S1 (Pons et al., 1992) are in contradiction with the findings of Zhang et al. (1996), showing that in higher primates the responses to non-nociceptive somatosensory stimuli in S2 are mostly unaffected by the reversible inactivation of S1 by cooling, thus indicating that the bulk of the inputs triggering the responses in S2 are, in fact, not relayed through S1.

Allison et al. (1989a,b) used direct intracranial recordings performed in patients to observe that non-nociceptive somatosensory stimuli elicit responses of earlier latency in S1 compared with S2. Similar findings have been made using source reconstruction and Granger causality analysis of magnetoencephalographic signals (Hari et al., 1993; Mima et al., 1998; Schnitzler et al., 1999; Inui et al., 2004; Ploner et al., 2009). Although these findings might suggest a serial processing of non-nociceptive somatosensory input from $S 1$ to S2, they could also be explained by either a slower response onset of S2 neurons (Trappenberg, 2002) or a slower conduction velocity of thalamocortical pathways to S2. Furthermore, they could also be explained by an incomplete sampling of the neural activity of S2 neurons because of the partial coverage of the S2 area by the intracranial electrodes (Allison et al., 1989a,b) or because of the poor sensitivity of magnetoencephalography to source currents that are deeply located or radially oriented relative to the skull surface (Lütkenhöner, 2003). Furthermore, because these different approaches did not allow sampling the activity within the thalamus, they could not examine directly the effective connectivity between the thalamus and $\mathrm{S} 1$ and between the thalamus and S2. Finally, it is important to highlight the fact that the magnetoencephalographic evidence suggesting a delayed response to non-nociceptive somatosensory input in S2 versus S1 has been contradicted by Karhu and Tesche (1999), who showed that the earliest response in S2 after non-nociceptive stimulation of the hand peaks at 20-30 ms after the onset of the stimulus (i.e., not later than the earliest peak of activity in S1), thus suggesting that S1 and S2 respond quasi-simultaneously to non-nociceptive somatosensory stimulation.

Importantly, the existence of serial projections of nonnociceptive somatosensory input from the thalamus to $\mathrm{S} 1$ and from
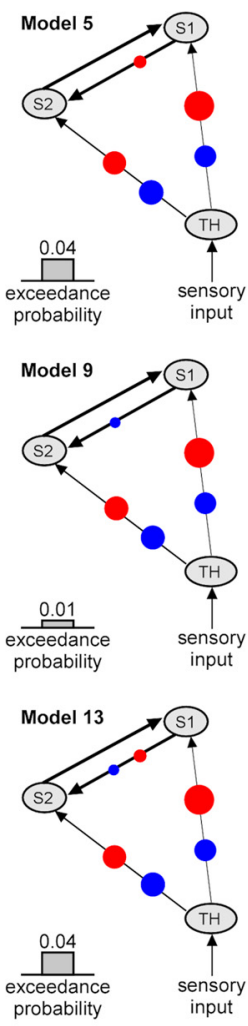
stimulation, respectively.
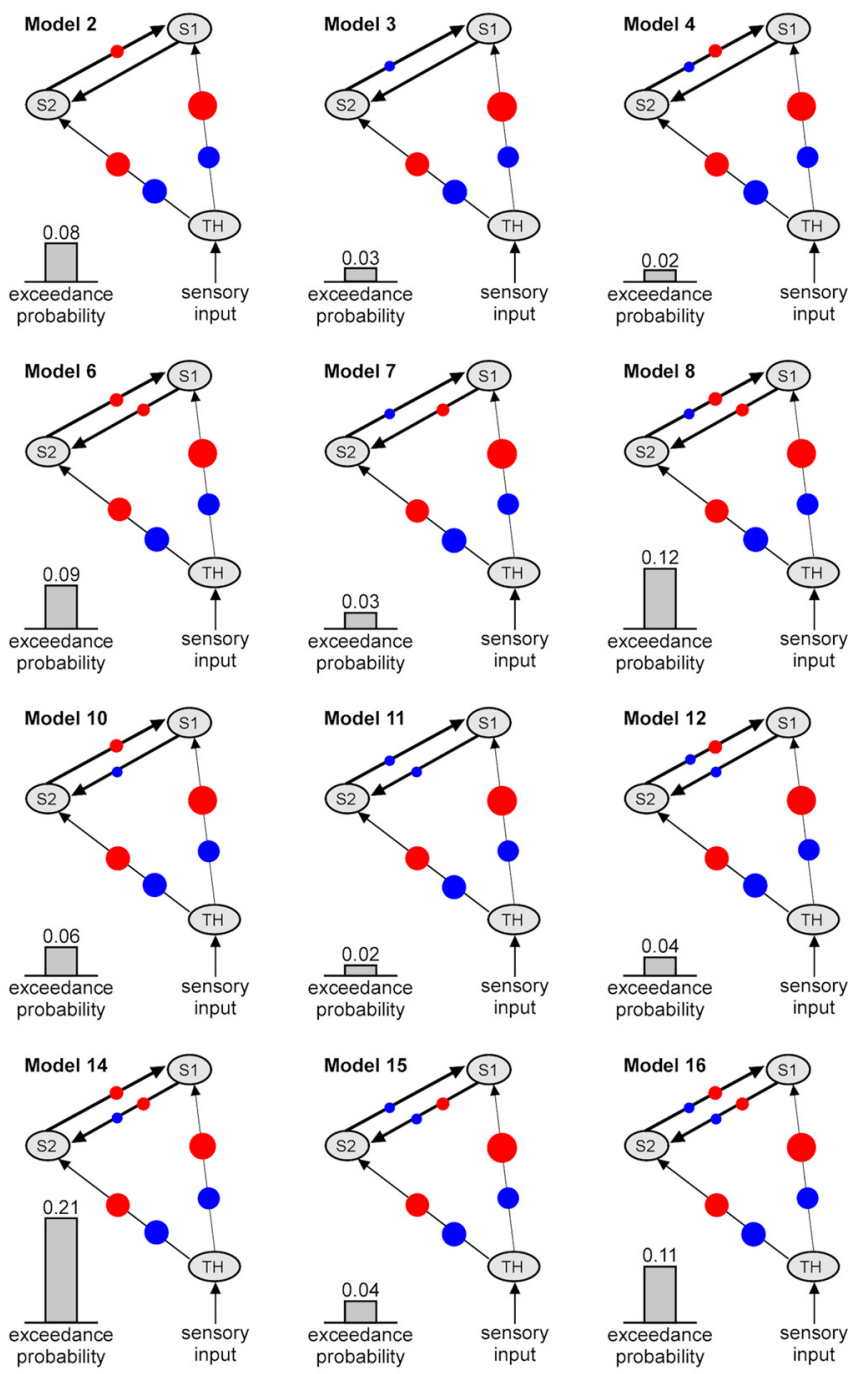

Figure 4. The estimated parameters and the exceedance probabilities of the 16 models belonging to the winning family $P$ (the family with the highest exceedance probability) (Fig. 3). These 16 single models share a common feature (i.e., that the connections wever, they differ in terms of how the connections between S1 and S2 are modulated. The black lines with arrows represent the the red and blue dots on each connection represents the magnitude of the modulatory effect of nociceptive or non-nociceptive

S1 to S2 does not exclude the coexistence of direct projections from the thalamus to S2. This has been suggested by Knecht et al. (1996), who showed that the perception of somatosensory qualities like vibration can be mostly unaffected in patients with lesions of the parietal cortex encompassing S1. Furthermore, the existence of parallel pathways to S2 would also explain why Pons et al. (1992) observed that the ablation of S1 does not abolish completely the responses to non-nociceptive somatosensory stimuli in S2. The idea of coexistent parallel and serial processing is also consistent with our finding of strong intrinsic connections between S1 and S2 (Fig. 4). However, the observations that the modulatory connections between the thalamus and S1 and between the thalamus and S2 are (1) of similar magnitude and (2) much stronger than the modulatory connections between S1 and S2, suggest that parallel 
processing dominates the information transmission between the thalamus, S1, and S2. Together, results from previous studies and our present findings indicate that the cortical processing of nonnociceptive inputs sampled using fMRI is not uniquely serial but involves mostly parallel pathways from the thalamus to S1 and from the thalamus to S2. It is important to note, however, that, as in most of previous studies investigating the serial versus parallel processing of tactile inputs in S1 and S2, we used electrical stimuli that activate all subpopulations of $\mathrm{A} \beta$ fibers in the nerve. Thus, we cannot rule out that the selective activation of a given subpopulation of $\mathrm{A} \beta$ fibers (e.g., fibers innervating Pacinian or Meissner's corpuscules) would result in a more serial organization of S1 and S2.

\section{Parallel processing of nociceptive input in S1 and S2}

Our results indicate that, similar to non-nociceptive somatosensory input, nociceptive somatosensory input is processed in parallel from the thalamus to $S 1$ and from the thalamus to S2 (Figs. 3, 4). The involvement of S1 and S2 in the cortical processing of nociceptive input in humans has been reported in a large number of studies (Ploner et al., 1999; Kanda et al., 2000; Porro, 2003; Youell et al., 2004; Apkarian et al., 2005; Plaghki and Mouraux, 2005; Tracey and Mantyh, 2007). However, only a few studies have examined explicitly whether the processing of nociceptive input in S1 and S2 is organized in parallel or serially. Using source reconstruction of magnetoencephalographic responses to nociceptive stimuli in humans, Ploner et al. (1999, 2009) observed that the responses hypothesized to originate from S1 and S2 had similar onset times $(\sim 130 \mathrm{~ms})$ and that the S2 activity was causally influenced by the S1 activity during non-nociceptive stimulation but not during nociceptive stimulation. This finding suggests a serial organization of non-nociceptive somatosensory processing from S1 to S2, and a parallel organization of nociceptive somatosensory processing in S1 and S2. Using a similar approach, Kanda et al. (2000) found that the onsets of the responses to nociceptive somatosensory stimuli in S1 and S2 were simultaneous in 7 of 12 participants. However, the interpretation of this observation remains speculative as it relies entirely on source analysis techniques whose reliability are inherently questionable because of (1) the infinite number of solutions to the inverse problem, (2) the possibly wrong assumptions required to define the forward model or to constrain the source models (e.g., number of dipoles and head model) (Lütkenhöner, 2003). Furthermore, and most importantly, the lack of sensitivity of magnetoencephalography to deeply located source currents (Lütkenhöner, 2003) makes it impossible to explore directly the actual relationships between responses in the thalamus and responses in S1 and S2. Because in the present study we used a completely different approach, both in terms of the method used to sample brain activity (i.e., hemodynamic BOLD fMRI signal) and in terms of the analysis technique (i.e., DCM and BMS), and because we compared directly the information flow between the thalamus, S1, and S2 using the BOLD responses elicited by nociceptive and non-nociceptive somatosensory input at single-subject level, our results provide strong and complementary support to the magnetoencephalographic evidence indicating that nociceptive inputs project in parallel from the thalamus to S1 and from the thalamus to S2.

In conclusion, our results indicate that the hierarchical organization of the thalamus-S1-S2 network involved in processing nociceptive and non-nociceptive stimuli is fundamentally similar. However, it is important to highlight that this conclusion is based on the recording of fMRI signals that integrate neural activity (1) of a large number of neurons and (2) over a timescale of the order of several seconds (Logothetis, 2008), and cannot thus provide details of the dynamic changes of this network at the finer spatial and temporal resolution attainable using invasive electrophysiology. Hence, our results cannot rule out that differences in the thalamus-S1-S2 network involved in processing nociceptive and non-nociceptive stimuli may exist at a smaller spatial or temporal scale.

\section{Notes}

Supplemental material for this article is available at http://iannettilab. webnode.com/products/supplementary-materials/. The reliability of our results was verified by four supplemental analyses: (1) we used two different ROI selection strategies, and (2) we added two additional brain areas to the model, the contralateral insula or the ipsilateral S2. The results of these additional analyses indicate that (1) our ROI selection based on conjunction map did not bias the results, and (2) the results were still valid when more complex model structures were tested. This material has not been peer reviewed.

\section{References}

Allison T, McCarthy G, Wood CC, Darcey TM, Spencer DD, Williamson PD (1989a) Human cortical potentials evoked by stimulation of the median nerve. I. Cytoarchitectonic areas generating short-latency activity. J Neurophysiol 62:694-710.

Allison T, McCarthy G, Wood CC, Williamson PD, Spencer DD (1989b) Human cortical potentials evoked by stimulation of the median nerve. II. Cytoarchitectonic areas generating long-latency activity. J Neurophysiol 62:711-722.

Apkarian AV, Bushnell MC, Treede RD, Zubieta JK (2005) Human brain mechanisms of pain perception and regulation in health and disease. Eur J Pain 9:463-484.

Bromm B, Treede RD (1984) Nerve fibre discharges, cerebral potentials and sensations induced by $\mathrm{CO}_{2}$ laser stimulation. Hum Neurobiol 3:33-40.

Büchel C, Friston KJ (1997) Modulation of connectivity in visual pathways by attention: cortical interactions evaluated with structural equation modelling and fMRI. Cereb Cortex 7:768-778.

Burgess PR, Perl ER (1967) Myelinated afferent fibres responding specifically to noxious stimulation of the skin. J Physiol 190:541-562.

Burton H, Carlson M (1986) Second somatic sensory cortical area (SII) in a prosimian primate, Galago crassicaudatus. J Comp Neurol 247:200-220.

Burton H, Jones EG (1976) The posterior thalamic region and its cortical projection in New World and Old World monkeys. J Comp Neurol 168:249-301.

Bushnell MC, Duncan GH, Hofbauer RK, Ha B, Chen JI, Carrier B (1999) Pain perception: is there a role for primary somatosensory cortex? Proc Natl Acad Sci U S A 96:7705-7709.

Caplan D, Moo L (2004) Cognitive conjunction and cognitive functions. Neuroimage 21:751-756.

Cohen LG, Bandinelli S, Sato S, Kufta C, Hallett M (1991) Attenuation in detection of somatosensory stimuli by transcranial magnetic stimulation. Electroencephalogr Clin Neurophysiol 81:366-376.

Daunizeau J, David O, Stephan KE (2009) Dynamic causal modelling: a critical review of the biophysical and statistical foundations. Neuroimage. Advance online publication. Retrieved May 19, 2011. doi: 10.1016/j.neuroimage.2009.11.062.

David O (2009) fMRI connectivity, meaning and empiricism: Comments on: Roebroeck et al. The identification of interacting networks in the brain using fMRI: model selection, causality and deconvolution. Neuroimage. Advance online publication. Retrieved May 19, 2011. doi: 10.1016/j.neuroimage.2009.09.073.

David O, Guillemain I, Saillet S, Reyt S, Deransart C, Segebarth C, Depaulis A (2008) Identifying neural drivers with functional MRI: an electrophysiological validation. PLoS Biol 6:2683-2697.

Friedman DP, Murray EA (1986) Thalamic connectivity of the second somatosensory area and neighboring somatosensory fields of the lateral sulcus of the macaque. J Comp Neurol 252:348-373.

Friedman DP, Jones EG, Burton H (1980) Representation pattern in the second somatic sensory area of the monkey cerebral cortex. J Comp Neurol 192:21-41.

Friston K (2009a) Causal modelling and brain connectivity in functional magnetic resonance imaging. PLoS Biol 7:e33. 
Friston K (2009b) Dynamic causal modeling and Granger causality. Comments on: The identification of interacting networks in the brain using fMRI: model selection, causality and deconvolution. Neuroimage. Advance online publication. Retrieved May 19, 2011. doi: 10.1016/j.neuroimage.2009.09.031.

Friston K, Mattout J, Trujillo-Barreto N, Ashburner J, Penny W (2007) Variational free energy and the Laplace approximation. Neuroimage 34:220-234.

Friston KJ, Buechel C, Fink GR, Morris J, Rolls E, Dolan RJ (1997) Psychophysiological and modulatory interactions in neuroimaging. Neuroimage 6:218-229.

Friston KJ, Holmes AP, Price CJ, Büchel C, Worsley KJ (1999) Multisubject fMRI studies and conjunction analyses. Neuroimage 10:385-396.

Friston KJ, Harrison L, Penny W (2003) Dynamic causal modelling. Neuroimage 19:1273-1302.

Friston KJ, Penny WD, Glaser DE (2005) Conjunction revisited. Neuroimage 25:661-667.

Garraghty PE, Florence SL, Tenhula WN, Kaas JH (1991) Parallel thalamic activation of the first and second somatosensory areas in prosimian primates and tree shrews. J Comp Neurol 311:289-299.

Gingold SI, Greenspan JD, Apkarian AV (1991) Anatomic evidence of nociceptive inputs to primary somatosensory cortex: relationship between spinothalamic terminals and thalamocortical cells in squirrel monkeys. J Comp Neurol 308:467-490.

Goebel R, Roebroeck A, Kim DS, Formisano E (2003) Investigating directed cortical interactions in time-resolved fMRI data using vector autoregressive modeling and Granger causality mapping. Magn Reson Imaging 21:1251-1261.

Hannula H, Neuvonen T, Savolainen P, Tukiainen T, Salonen O, Carlson S, Pertovaara A (2008) Navigated transcranial magnetic stimulation of the primary somatosensory cortex impairs perceptual processing of tactile temporal discrimination. Neurosci Lett 437:144-147.

Hari R, Karhu J, Hämäläinen M, Knuutila J, Salonen O, Sams M, Vilkman V (1993) Functional organization of the human first and second somatosensory cortices: a neuromagnetic study. Eur J Neurosci 5:724-734.

Inui K, Wang X, Tamura Y, Kaneoke Y, Kakigi R (2004) Serial processing in the human somatosensory system. Cereb Cortex 14:851-857.

Iwamura Y (1998) Hierarchical somatosensory processing. Curr Opin Neurobiol 8:522-528.

Jones EG (1998) Viewpoint: the core and matrix of thalamic organization. Neuroscience 85:331-345.

Jones EG, Leavitt RY (1974) Retrograde axonal transport and the demonstration of non-specific projections to the cerebral cortex and striatum from thalamic intralaminar nuclei in the rat, cat and monkey. J Comp Neurol 154:349-377.

Kanda M, Nagamine T, Ikeda A, Ohara S, Kunieda T, Fujiwara N, Yazawa S, Sawamoto N, Matsumoto R, Taki W, Shibasaki H (2000) Primary somatosensory cortex is actively involved in pain processing in human. Brain Res 853:282-289.

Kandel ER, Schwartz JH, Jessell TM (2010) Principles of neural science, Ed 5. New York, London: McGraw-Hill.

Karhu J, Tesche CD (1999) Simultaneous early processing of sensory input in human primary (SI) and secondary (SII) somatosensory cortices. J Neurophysiol 81:2017-2025.

Kayser C, Petkov CI, Lippert M, Logothetis NK (2005) Mechanisms for allocating auditory attention: an auditory saliency map. Curr Biol 15:1943-1947.

Kiebel SJ, Klöppel S, Weiskopf N, Friston KJ (2007) Dynamic causal modeling: a generative model of slice timing in fMRI. Neuroimage 34:1487-1496.

Knecht S, Kunesch E, Schnitzler A (1996) Parallel and serial processing of haptic information in man: effects of parietal lesions on sensorimotor hand function. Neuropsychologia 34:669-687.

Knecht S, Ellger T, Breitenstein C, Bernd Ringelstein E, Henningsen $\mathrm{H}$ (2003) Changing cortical excitability with low-frequency transcranial magnetic stimulation can induce sustained disruption of tactile perception. Biol Psychiatry 53:175-179.

Krubitzer LA, Kaas JH (1992) The somatosensory thalamus of monkeys: cortical connections and a redefinition of nuclei in marmosets. J Comp Neurol 319:123-140.

Leff AP, Schofield TM, Stephan KE, Crinion JT, Friston KJ, Price CJ (2008) The cortical dynamics of intelligible speech. J Neurosci 28:13209-13215.
Logothetis NK (2008) What we can do and what we cannot do with fMRI. Nature 453:869-878.

Lütkenhöner B (2003) Magnetoencephalography and its Achilles' heel. J Physiol Paris 97:641-658.

Marreiros AC, Kiebel SJ, Friston KJ (2008) Dynamic causal modelling for fMRI: a two-state model. Neuroimage 39:269-278.

Mayhew SD, Dirckx SG, Niazy RK, Iannetti GD, Wise RG (2010) EEG signatures of auditory activity correlate with simultaneously recorded fMRI responses in humans. Neuroimage 49:849-864.

McIntosh AR, Gonzalez-Lima F (1994) Structural equation modeling and its application to network analysis in functional brain imaging. Hum Brain Mapp 2:2-22.

Mima T, Nagamine T, Nakamura K, Shibasaki H (1998) Attention modulates both primary and second somatosensory cortical activities in humans: a magnetoencephalographic study. J Neurophysiol 80:2215-2221.

Mountcastle VB (2005) The sensory hand: neural mechanisms of somatic sensation. Cambridge, MA: Harvard UP.

Mouraux A, Iannetti GD (2009) Nociceptive laser-evoked brain potentials do not reflect nociceptive-specific neural activity. J Neurophysiol 101:3258-3269.

Mouraux A, Iannetti GD, Plaghki L (2010) Low intensity intra-epidermal electrical stimulation can activate Adelta-nociceptors selectively. Pain 150:199-207.

Mouraux A, Diukova A, Lee MC, Wise RG, Iannetti GD (2011) A multisensory investigation of the functional significance of the "pain matrix." Neuroimage 54:2237-2249.

Nichols T, Brett M, Andersson J, Wager T, Poline JB (2005) Valid conjunction inference with the minimum statistic. Neuroimage 25:653-660.

Penny WD, Stephan KE, Mechelli A, Friston KJ (2004) Comparing dynamic causal models. Neuroimage 22:1157-1172.

Penny WD, Stephan KE, Daunizeau J, Rosa MJ, Friston KJ, Schofield TM, Leff AP (2010) Comparing families of dynamic causal models. PLoS Comput Biol 6:e1000709.

Plaghki L, Mouraux A (2005) EEG and laser stimulation as tools for pain research. Curr Opin Investig Drugs 6:58-64.

Ploner M, Schmitz F, Freund HJ, Schnitzler A (1999) Parallel activation of primary and secondary somatosensory cortices in human pain processing. J Neurophysiol 81:3100-3104.

Ploner M, Schoffelen JM, Schnitzler A, Gross J (2009) Functional integration within the human pain system as revealed by Granger causality. Hum Brain Mapp 30:4025-4032.

Pons TP, Kaas JH (1986) Corticocortical connections of area 2 of somatosensory cortex in macaque monkeys: a correlative anatomical and electrophysiological study. J Comp Neurol 248:313-335.

Pons TP, Garraghty PE, Mishkin M (1992) Serial and parallel processing of tactual information in somatosensory cortex of rhesus monkeys. J Neurophysiol 68:518-527.

Porro CA (2003) Functional imaging and pain: behavior, perception, and modulation. Neuroscientist 9:354-369.

Price CJ, Friston KJ (1997) Cognitive conjunction: a new approach to brain activation experiments. Neuroimage 5:261-270.

Roebroeck A, Formisano E, Goebel R (2009a) The identification of interacting networks in the brain using fMRI: model selection, causality and deconvolution. Neuroimage. Advance online publication. Retrieved May 19, 2011. doi: 10.1016/j.neuroimage.2009.09.036.

Roebroeck A, Formisano E, Goebel R (2009b) Reply to Friston and David after comments on: The identification of interacting networks in the brain using fMRI: model selection, causality and deconvolution. Neuroimage. Advance online publication. Retrieved May 19, 2011. doi: 10.1016/j.neuroimage.2009.10.077.

Rowe MJ, Turman AB, Murray GM, Zhang HQ (1996) Parallel organization of somatosensory cortical areas I and II for tactile processing. Clin Exp Pharmacol Physiol 23:931-938.

Schnitzler A, Volkmann J, Enck P, Frieling T, Witte OW, Freund HJ (1999) Different cortical organization of visceral and somatic sensation in humans. Eur J Neurosci 11:305-315.

Schuyler B, Ollinger JM, Oakes TR, Johnstone T, Davidson RJ (2010) Dynamic causal modeling applied to fMRI data shows high reliability. Neuroimage 49:603-611.

Seghier ML, Price CJ (2010) Reading aloud boosts connectivity through the putamen. Cereb Cortex 20:570-582.

Shi T, Stevens RT, Tessier J, Apkarian AV (1993) Spinothalamocortical in- 
puts nonpreferentially innervate the superficial and deep cortical layers of SI. Neurosci Lett 160:209-213.

Stephan KE, Marshall JC, Penny WD, Friston KJ, Fink GR (2007) Interhemispheric integration of visual processing during task-driven lateralization. J Neurosci 27:3512-3522.

Stephan KE, Kasper L, Harrison LM, Daunizeau J, den Ouden HE, Breakspear M, Friston KJ (2008) Nonlinear dynamic causal models for fMRI. Neuroimage 42:649-662.

Stephan KE, Penny WD, Daunizeau J, Moran RJ, Friston KJ (2009) Bayesian model selection for group studies. Neuroimage 46:1004-1017.

Stephan KE, Penny WD, Moran RJ, den Ouden HE, Daunizeau J, Friston KJ (2010) Ten simple rules for dynamic causal modeling. Neuroimage 49:3099-3109.

Stevens RT, London SM, Apkarian AV (1993) Spinothalamocortical projections to the secondary somatosensory cortex (SII) in squirrel monkey. Brain Res 631:241-246.

Tracey I, Mantyh PW (2007) The cerebral signature for pain perception and its modulation. Neuron 55:377-391.
Trappenberg TP (2002) Fundamentals of computational neuroscience. Oxford, New York: Oxford UP.

Turman AB, Ferrington DG, Ghosh S, Morley JW, Rowe MJ (1992) Parallel processing of tactile information in the cerebral cortex of the cat: effect of reversible inactivation of SI on responsiveness of SII neurons. J Neurophysiol 67:411-429.

Youell PD, Wise RG, Bentley DE, Dickinson MR, King TA, Tracey I, Jones AK (2004) Lateralisation of nociceptive processing in the human brain: a functional magnetic resonance imaging study. Neuroimage 23:10681077.

Zhang HQ, Murray GM, Turman AB, Mackie PD, Coleman GT, Rowe M] (1996) Parallel processing in cerebral cortex of the marmoset monkey: effect of reversible SI inactivation on tactile responses in SII. J Neurophysiol 76:3633-3655.

Zhang HQ, Zachariah MK, Coleman GT, Rowe MJ (2001) Hierarchical equivalence of somatosensory areas I and II for tactile processing in the cerebral cortex of the marmoset monkey. J Neurophysiol 85:18231835. 